\title{
Acute hemodynamic effects of inhaled sodium nitrite in pulmonary hypertension associated with heart failure with preserved ejection fraction
}

\author{
Marc A. Simon,, 1,2,3,4 Rebecca R. Vanderpool, ,,3 Mehdi Nouraie,, ${ }^{2,4}$ Timothy N. Bachman,, 2,3 \\ Pamela M. White, ${ }^{4}$ Masataka Sugahara, ${ }^{1}$ John Gorcsan III, ${ }^{1}$ Ed L. Parsley, ${ }^{5}$ and Mark T. Gladwin ${ }^{2,4}$ \\ ${ }^{1}$ Heart and Vascular Institute, ${ }^{2}$ Pittsburgh Heart, Lung, Blood and Vascular Medicine Institute, ${ }^{3}$ Department of \\ Bioengineering, and ${ }^{4}$ Division of Pulmonary, Allergy and Critical Care Medicine, Department of Medicine, University of \\ Pittsburgh, Pittsburgh, Pennsylvania, USA. ${ }^{5}$ Mast Therapeutics, Inc, San Diego, California, USA.
}

BACKGROUND. Pulmonary hypertension (PH) is associated with poor outcomes, yet specific treatments only exist for a small subset of patients. The most common form of $\mathrm{PH}$ is that associated with left heart disease (Group 2), for which there is no approved therapy. Nitrite has shown efficacy in preclinical animal models of Group 1 and $2 \mathrm{PH}$, as well as in patients with left heart failure with preserved ejection fraction (HFpEF). We evaluated the safety and efficacy of a potentially novel inhaled formulation of nitrite in PH-HFpEF patients as compared with Group 1 and $3 \mathrm{PH}$.

METHODS. Cardiopulmonary hemodynamics were recorded after acute administration of inhaled nitrite at 2 doses, 45 and $90 \mathrm{mg}$. Safety endpoints included change in systemic blood pressure and methemoglobin levels. Responses were also compared with those administered inhaled nitric oxide.

RESULTS. Thirty-six patients were enrolled (10 PH-HFpEF, 20 Group 1 pulmonary arterial hypertension patients on background PH-specific therapy, and 6 Group $3 \mathrm{PH}$ ). Drug administration was well tolerated. Nitrite inhalation significantly lowered pulmonary, right atrial, and pulmonary capillary wedge pressures, most pronounced in patients with PH-HFpEF. There was a modest decrease in cardiac output and systemic blood pressure. Pulmonary vascular resistance decreased only in Group $3 \mathrm{PH}$ patients. There was substantial increase in pulmonary artery compliance, most pronounced in patients with PH-HFpEF.

CONCLUSIONS. Inhaled nitrite is safe in PH patients and may be efficacious in PH-HFpEF and Group $3 \mathrm{PH}$ primarily via improvements in left and right ventricular filling pressures and pulmonary artery compliance. The lack of change in pulmonary vascular resistance likely may limit efficacy for Group 1 patients.

TRIAL REGISTRATION. ClinicalTrials.gov NCT01431313

Conflict of interest: M.A. Simon has received consultancy fees from United Therapeutics and Gilead. E.L. Parsley is an employee of Mast Therapeutics, Inc. M.T. Cladwin is a coinventor of awarded patent (number 9,387,224) for nitrite therapy for cardiovascular indications. M.T. Cladwin receives sponsored research support for the inhaled nitrite clinical trial (note: M.T.C. does not receive consulting fees from Mast Therapeutics).

Submitted: August 3, 2016 Accepted: September 29, 2016 Published: November 3, 2016

Reference information: JCI Insight. 2016;1(18):e89620. doi:10.1172/jici.nsight.89620.

FUNDING. This work was supported in part by the NIH grants 2P01HL103455 (to MAS and MTG), R01HL098032 (to MTC), and R01HL096973 (to MTC), and Mast Therapeutics, Inc.

\section{Introduction}

Pulmonary hypertension $(\mathrm{PH})$ is comprised of a highly heterogeneous group of diseases that uniformly results in functional impairment and poor outcomes. Specific treatment has been limited to a small subset of patients with World Health Organization (WHO) Group 1 pulmonary arterial hypertension (PAH), defined hemodynamically by a mean pulmonary artery pressure greater than or equal to $25 \mathrm{mmHg}$ and a pulmonary capillary wedge pressure less than or equal to $15 \mathrm{mmHg}$, and WHO Group 4 chronic thromboembolic pulmonary hypertension. While there have been some studies in more prevalent types of $\mathrm{PH}$, such as WHO Group 2 ( $\mathrm{PH}$ due to left heart disease, defined hemodynamically by a mean pulmonary artery pressure greater than or equal to $25 \mathrm{mmHg}$ and a pulmonary capillary wedge pressure greater than 
$15 \mathrm{mmHg}$ ) and WHO Group 3 (PH due to lung disease and/or hypoxia), to date there are no approved treatments for these patients.

$\mathrm{PH}$ in the setting of left heart disease is the most common form of $\mathrm{PH}$ with a prevalence between $23 \%$ and $83 \%$ of patients with heart failure with preserved ejection fraction (HFpEF) $(1,2)$. Chronic pulmonary venous congestion results in elevated pulmonary arterial pressure, eventually resulting in vascular remodeling with decreased nitric oxide-dependent (NO-dependent) vasodilation and increased endothelin expression (1, 2). Outcomes are worse when $\mathrm{PH}$ complicates left heart disease and there are no specific treatments (1-4). Further, in patients with left heart disease, therapies indicated for PAH, such as prostacyclins and endothelin receptor antagonists have been unsuccessful, while phosphodiesterase type 5 inhibitors have met with mixed results (5-9). Therefore, a great need exists to develop targeted therapies and to identify the patient population that can benefit. The exact definition of $\mathrm{PH}$ in the setting of left heart disease is still debated, but includes at least the criteria of a mean pulmonary artery pressure greater than or equal to $25 \mathrm{mmHg}$ and a pulmonary capillary wedge pressure greater than or equal to $15 \mathrm{mmHg}(10)$. Additional hemodynamic criteria of $\mathrm{PH}$ in the setting of left heart disease, along with how such criteria affect prevalence and outcomes, continue to be debated, with the ultimate goal to identify a phenotype amenable to specific targeted therapies (1, 2, 10-15).

The NO signaling pathway has been targeted therapeutically in Group 1 PAH, with FDA approval of phosphodiesterase 5 inhibitors and small molecular stimulators of the soluble guanylate cyclase enzyme. NO gas and drugs that enhance NO production in vivo, such as L-arginine, have been proposed as potential therapies (16). However, NO has a very short half-life in blood $(<2$ milliseconds) and eNOS is uncoupled in conditions of hypoxia, inflammation, and oxidant stress. These limitations may be obviated by the anion nitrite $\left(\mathrm{NO}_{2}^{-}\right)$, which has a longer half-life (about 40 minutes) and is reduced to NO, particularly during hypoxia and acidosis $(17,18)$. Exogenously administered nitrite is a vasodilator and in preclinical models of $\mathrm{PH}$ has shown efficacy in reducing pulmonary pressures and preventing right ventricular hypertrophy (1921). More recent work has demonstrated that acute IV administration of nitrite improves exercise capacity in patients with $\mathrm{HFpEF}$, a group at high risk for secondary (Group 2) $\mathrm{PH}$ (22). In a recent study by our group, chronic therapy with oral nitrite improved $\mathrm{PH}$ in a rat model of severe metabolic syndrome and $\mathrm{PH}$ associated with HFpEF (PH-HFpEF) (23).

We therefore sought to evaluate in a phase IIa safety and efficacy trial the acute effects of an inhaled formulation of nitrite on cardiopulmonary hemodynamics in patients with $\mathrm{PH}-\mathrm{HFpEF}$ as compared with WHO Group $1 \mathrm{PAH}$ or Group $3 \mathrm{PH}$.

\section{Results}

Safety of inhaled sodium nitrite. Ten patients with $\mathrm{PH}-\mathrm{HFpEF}, 20$ patients with Group $1 \mathrm{PAH}$, and 6 patients with Group $3 \mathrm{PH}$ were studied (Figure 1). Clinical characteristics are presented in Table 1, baseline echocardiographic data in Table 2, and baseline hemodynamics in Table 3 and Supplemental Table 1. The safety stopping criteria consisted of the following: decrease in systemic systolic blood pressure greater than $40 \mathrm{mmHg}$, decrease in peripheral oxygen saturation greater than $10 \%$, methemoglobin level greater than $5 \%$, and severe bronchospasm or dyspnea. Drug administration appeared to be well tolerated in all patients, even in Group 1 patients on extensive background vasodilator therapy (Table 1). One patient (PH-HFpEF) did not receive the second dose of $90 \mathrm{mg}$ due to an asymptomatic transient decrease in systemic systolic blood pressure that met the prospectively defined stopping criteria but returned to baseline within 5 minutes without intervention. A second patient with Group $2 \mathrm{PH}-\mathrm{HFpEF}$ did not receive the second dose of $90 \mathrm{mg}$ due to musculoskeletal back pain during the case and was unable to continue through to the end of the protocol. There were no significant decreases in peripheral oxygen saturation nor increases in methemoglobin levels above the stopping criteria of $5 \%$. The only side effect reported during drug administration was an occasional, self-limited cough that required no intervention.

Hemodynamic effects on inhaled nitrite in patients with $P H-H F p E F$. In the 10 patients enrolled with $\mathrm{PH}-\mathrm{HFpEF}$, acute administration of inhaled sodium nitrite resulted in significant overall decreases in right atrial pressure, pulmonary capillary wedge pressure, right ventricular systolic and diastolic, and pulmonary artery systolic, diastolic, and mean pressures (Figure 2, and Supplemental Table 2. Of note, by the primary mixed-effect model analysis, pulmonary capillary wedge pressure and mean pulmonary artery pressure markedly decreased by $7.5 \mathrm{mmHg}(95 \% \mathrm{CI}-9.0$ to -6.0$)$ and $7.9 \mathrm{mmHg}(95 \% \mathrm{CI}-9.4$ to -6.3$)$, respectively (baseline median values 18 and $34 \mathrm{mmHg}$, respectively). With significant lowering of all pressures, there was no significant change in transpulmonary gradient and a modest but significant increase in pulmonary vascular 
140

Subjects screened for eligibility

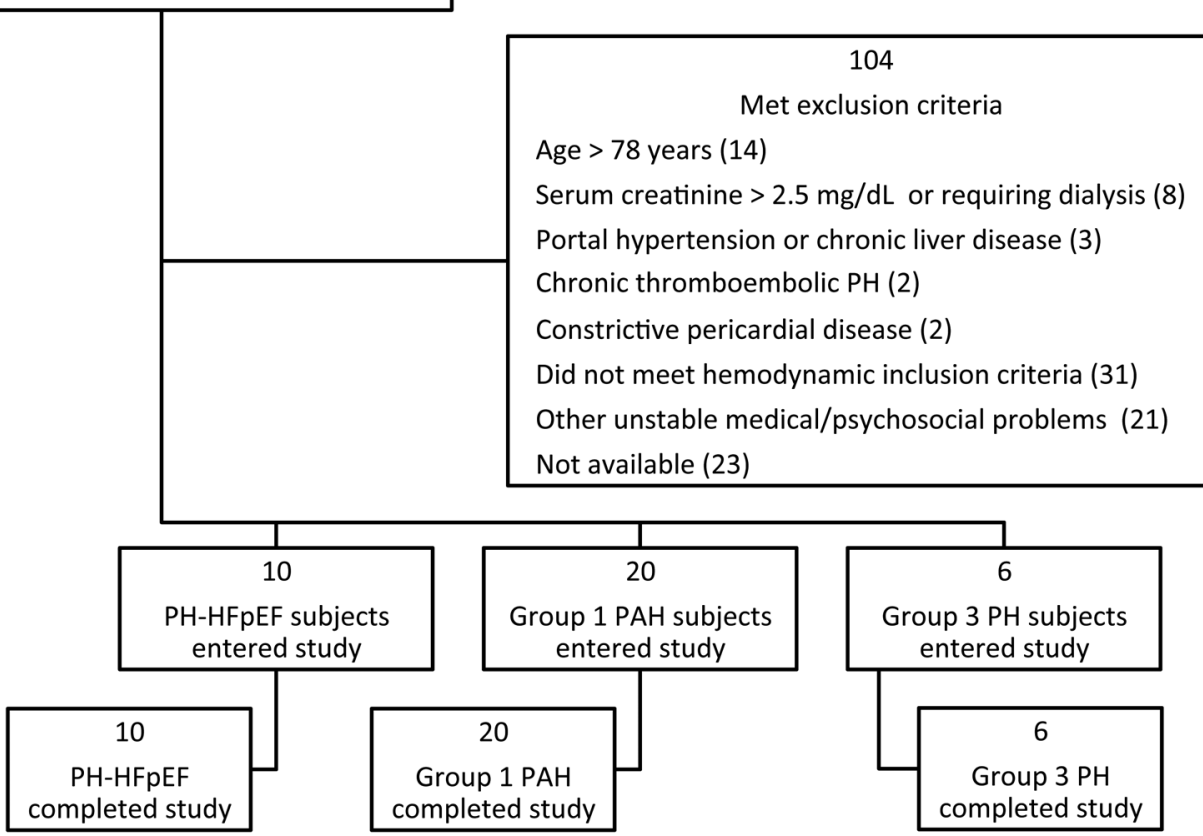

Figure 1. Screening and enrollment flow chart. $\mathrm{PH}$, pulmonary hypertension; PH-HFpEF, pulmonary hypertension associated with heart failure with preserved ejection fraction; $\mathrm{PAH}$, pulmonary arterial hypertension.

resistance (PVR). Cardiac index decreased slightly but significantly $(-0.2 \quad 1 / \mathrm{min} /$ $\mathrm{m}^{2}, 95 \% \mathrm{CI}-0.3$ to $-0.07, P$ $=0.003)$ as did mean arterial pressure $(-5.4 \mathrm{mmHg}$, 95\% CI -9.6 to $-1.1, P=$ 0.013 ), although there was no significant increase in heart rate; systemic vascular resistance very modestly increased $(+0.10 \mathrm{mmHg} / 1 /$ $\min , 95 \% \mathrm{CI}+0.03$ to +0.18 ; $P=0.008)$, probably related to the decrease in cardiac output, and there was no change in systemic arterial compliance. Pulmonary artery compliance (Cpa) increased by $35 \%(+0.97 \mathrm{ml} / \mathrm{mmHg}, 95 \% \mathrm{CI}+0.25$ to $+1.68 ; P=0.008)$.

Further analysis of the dose effect of nitrite ( $45 \mathrm{mg}$ vs. $90 \mathrm{mg}$ dose) found that most hemodynamics were affected in a dose-dependent manner, with the exception of $\mathrm{Cpa}$. There was a significant dose effect of inhaled sodium nitrite on right atrial pressure $(-6.1$ and $-8.1 \mathrm{mmHg}$ with 45 and $90 \mathrm{mg}$ doses, respectively, $P<0.001)$, mean pulmonary artery pressure $(-7.6$ and $8.2 \mathrm{mmHg}$ with 45 and $90 \mathrm{mg}$ doses, respectively, $P$ $<0.001$ ), and pulmonary capillary wedge pressure $(-6.9$ and $-8.4 \mathrm{mmHg}$ with 45 and $90 \mathrm{mg}$ doses, respectively, $P<0.001$; Supplemental Table 3$)$. Cardiac index decreased in a dose-dependent manner $(-0.2$ and $-0.31 / \mathrm{min} / \mathrm{m}^{2}$ with 45 and $90 \mathrm{mg}$ doses, respectively, $\left.P<0.001\right)$. The increase in Cpa was not dose related. Inhaled sodium nitrite did have a dose-dependent effect on mean arterial pressure $(-3.8$ and $-7.6 \mathrm{mmHg}$ with 45 and $90 \mathrm{mg}$ doses, respectively, $P<0.001$ )

Hemodynamic effects on inhaled nitrite in patients with Group 1 PAH. Inhaled sodium nitrite had an overall significant effect in lowering right atrial pressure, pulmonary capillary wedge pressure, right ventricular diastolic, and pulmonary arterial systolic pressures (Figure 3; detailed in Supplemental Table 2). There was no significant effect on the transpulmonary gradient, cardiac index, heart rate, or systemic vascular resistance. There was a modest but significant increase in the PVR, secondary to symmetric decreases in both the mean pulmonary artery pressure and pulmonary capillary wedge pressure, with no change in cardiac output. There was no significant change in Cpa or peripheral oxygen saturation. A decrease in systemic blood pressures $(-5.1 \mathrm{mmHg}$ mean arterial pressure, $95 \% \mathrm{CI}-7.6$ to $-2.6, P<0.001)$ was observed.

Analysis of the dose effect of nitrite $(45 \mathrm{mg}$ vs. $90 \mathrm{mg}$ ) revealed a significant dose-related decrease in right atrial pressure $(-1.9$ and $-2.5 \mathrm{mmHg}$ with 45 and $90 \mathrm{mg}$ doses, respectively, $P<0.001)$ and pulmonary capillary wedge pressure $(-2.0$ and $-2.9 \mathrm{mmHg}$ with 45 and $90 \mathrm{mg}$ doses, respectively, $P<0.001$, Supplemental Table 3). There was a modest but significant dose-related increase in transpulmonary gradient $(+1.3$ and $+2.3 \mathrm{mmHg}$ with 45 and $90 \mathrm{mg}$ doses, respectively, $P=0.008)$ and PVR $(+0.09$ and +0.1 Woods units with 45 and $90 \mathrm{mg}$ doses, respectively, $P=0.002)$, as well as dose-related decrease in mean arterial pressure ( -4.6 and $-5.6 \mathrm{mmHg}$ with 45 and $90 \mathrm{mg}$ doses, respectively, $P<0.001$ ).

Hemodynamic effects on inhaled nitrite in patients with Group 3 PH. In 6 patients enrolled with Group 3 $\mathrm{PH}$ ( $\mathrm{PH}$ due to lung disease and/or hypoxia), inhaled nitrite caused an acute decrease in right atrial pressure, pulmonary capillary wedge pressure, right ventricular systolic, pulmonary artery systolic, diastolic, 
Table 1. Clinical characteristics of the cohorts

\begin{tabular}{|c|c|c|c|}
\hline & PH-HFpEF $n=10$ & Group 1 PAH $n=20$ & Group $3 \mathrm{PH} n=6$ \\
\hline Female gender, $n(\%)$ & $6(60 \%)$ & $13(65 \%)$ & $2(33 \%)$ \\
\hline \multirow[t]{3}{*}{ Etiology $(n)$} & HFpEF (10) & Idiopathic PAH (10) & ILD (3) \\
\hline & & SSC (8) & COPD (3) \\
\hline & & HIV (1) & \\
\hline CAD & $3(30 \%)$ & $4(20 \%)$ & $2(33 \%)$ \\
\hline Hypertension & $10(100 \%)$ & $6(30 \%)$ & $5(83 \%)$ \\
\hline Diabetes & $9(90 \%)$ & $5(25 \%)$ & $3(50 \%)$ \\
\hline Hyperlipidemia & $10(100 \%)$ & $4(20 \%)$ & $5(83 \%)$ \\
\hline \multicolumn{4}{|c|}{ PH medications } \\
\hline ACEi or ARB & $7(70 \%)$ & $4(20 \%)$ & $3(50 \%)$ \\
\hline Beta blocker & $10(100 \%)$ & $11(55 \%)$ & $4(20 \%)$ \\
\hline Statin & $8(80 \%)$ & $4(67 \%)$ & $5(83 \%)$ \\
\hline Diuretic & $10(100 \%)$ & $17(85 \%)$ & $6(100 \%)$ \\
\hline WHO Functional Class & $3.00 \pm 0.47$ & $2.55 \pm 0.51$ & $3.00 \pm 0.00$ \\
\hline I/II/III/IV & $0 / 1 / 8 / 1$ & $0 / 9 / 11 / 0$ & $0 / 0 / 6 / 0$ \\
\hline NT-Pro-BNP (pg/ml) & $195(122-682)$ & $540(145-928)$ & $83(23-1831)$ \\
\hline Methemoglobin (\%) & $0.8(0.6-1.1)$ & $0.8(0.6-1.0)$ & $1.0(0.9-1.3)$ \\
\hline \multicolumn{4}{|c|}{$\begin{array}{l}\text { Data displayed as mean } \pm \text { standard deviation, median (interquartile range), or } n(\%) \text {. CAD, coronary artery disease; PH, pulmonary hypertension; PH-HFpE } \\
\text { pulmonary hypertension associated with heart failure with preserved ejection fraction; PAH, pulmonary arterial hypertension; SSC, systemic sclerosis; } \\
\text { HIV, human immunodeficiency virus; CHD, congenital heart disease; ILD, interstitial lung disease; COPD, chronic obstructive pulmonary disease; PDE5i, } \\
\text { phosphodiesterase type } 5 \text { inhibitor; ERA, endothelin receptor antagonist; ACEi, angiotensin-converting enzyme inhibitor; ARB, angiotensin receptor } \\
\text { blocker; WHO, World Health Organization; NT-Pro-BNP, N-terminal pro-B-type natriuretic peptide. }\end{array}$} \\
\hline
\end{tabular}

and mean pressures (Figure 4 and Supplemental Table 2). There was a modest but significant decrease in PVR. There was no overall change in cardiac index. Cpa significantly increased $(+0.39 \mathrm{ml} / \mathrm{mmHg}, 95 \%$ $\mathrm{CI}+0.11$ to $+0.67 ; P=0.006)$. There was a significant decrease in mean arterial pressure $(-7.5 \mathrm{mmHg}$ in mean arterial pressure, $95 \% \mathrm{CI}-11.0$ to $-4.0 ; P<0.001)$ and systemic vascular resistance, with an increase in systemic arterial compliance $(+0.35 \mathrm{ml} / \mathrm{mmHg}, 95 \% \mathrm{CI}+0.13$ to $+0.58 ; P=0.002)$ but no change in heart rate or peripheral oxygen saturation.

A significant dose effect of nitrite ( $45 \mathrm{mg}$ vs. $90 \mathrm{mg}$ ) was noted for right atrial pressure $(-1.3$ and -2.7 mmHg with 45 and $90 \mathrm{mg}$ doses, respectively, $P<0.001)$, pulmonary capillary wedge pressure $(-3.1$ and $-5.7 \mathrm{mmHg}$ with 45 and $90 \mathrm{mg}$ doses, respectively, $P<0.001)$, right ventricular $(-1.9$ and $-4.8 \mathrm{mmHg}$ with 45 and $90 \mathrm{mg}$ doses, respectively, $P=0.003)$ and pulmonary artery diastolic $(-3.8$ and $-5.1 \mathrm{mmHg}$ with 45 and $90 \mathrm{mg}$ doses, respectively, $P<0.001)$ and mean pressures $(-4.4$ and $-5.3 \mathrm{mmHg}$ with 45 and $90 \mathrm{mg}$ doses, respectively, $P<0.001$, Supplemental Table 3$)$. There was a dose-related increase in cardiac index $\left(+0.1\right.$ and $+0.41 / \mathrm{min} / \mathrm{m}^{2}$ with 45 and $90 \mathrm{mg}$ doses, respectively, $\left.P=0.002\right)$, but no dose-related effect on PVR. Cpa increased in a dose-dependent manner $(+0.36$ and $+0.43 \mathrm{ml} / \mathrm{mmHg}$, with 45 and $90 \mathrm{mg}$ doses, respectively, $P=0.006)$. The decrease in mean arterial pressure was also dose related $(-7.2$ and $-7.9 \mathrm{ml} /$ $\mathrm{mmHg}$, with 45 and $90 \mathrm{mg}$ doses, respectively, $P=0.002$ ), as was the increase in methemoglobin levels.

Comparison of effects of inhaled sodium nitrite between different $\mathrm{PH}$ groups. The greatest decrease in pulmonary capillary wedge pressure was in PH-HFpEF. Right atrial pressure, pulmonary capillary wedge pressure, right ventricular, and pulmonary artery pressures all decreased more in PH-HFpEF (Supplemental Table 2) as compared with Group $1 \mathrm{PAH}$ patients. There was also a greater effect on pulmonary artery diastolic and mean pressures observed in Group 3 versus Group 1. PH-HFpEF and Group 3 PH had simi- 

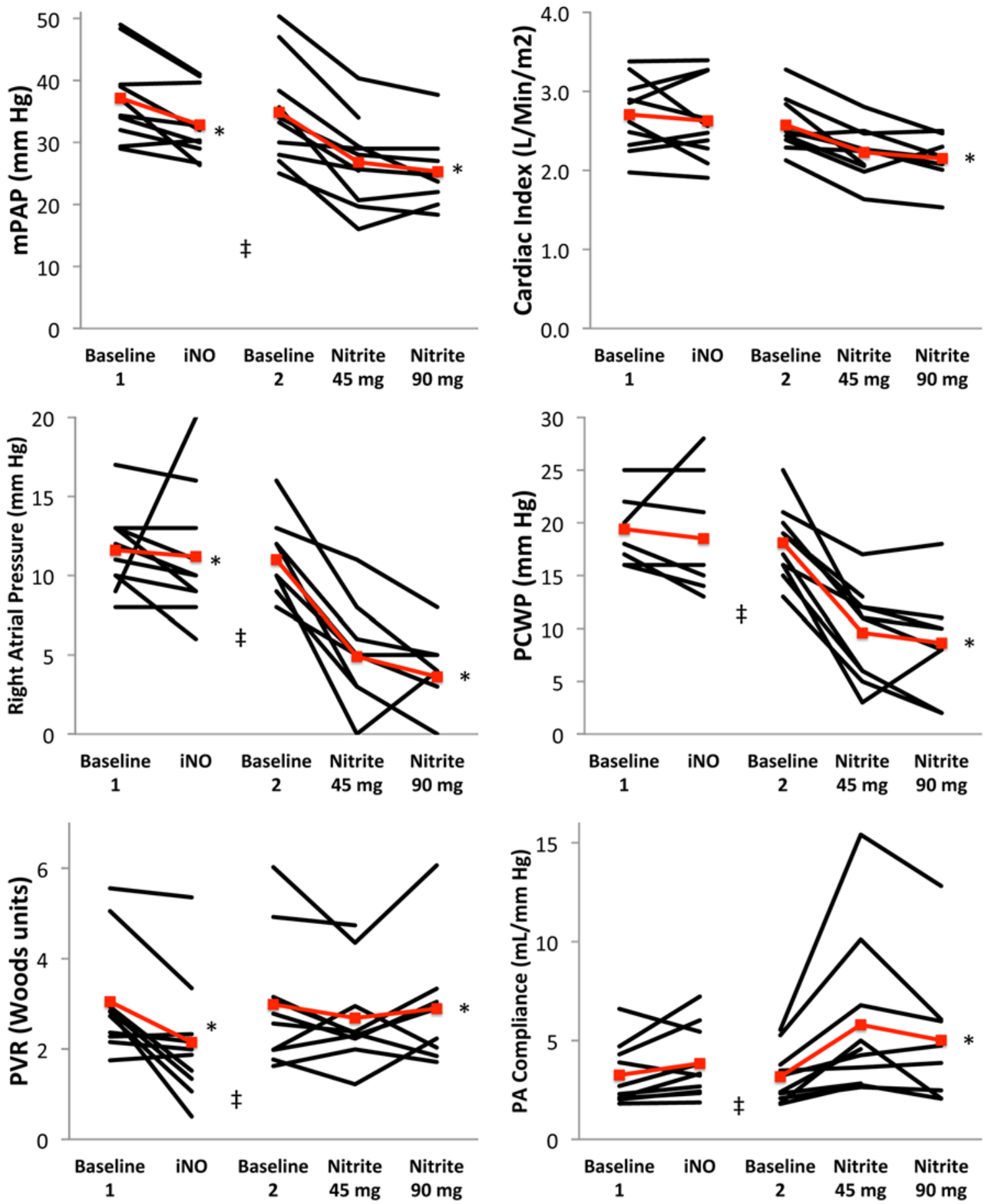

Figure 2. Effect of inhaled nitric oxide and aerosolized sodium nitrite on PH-HFpEF patients. mPAP, mean pulmonary artery pressure; PA, pulmonary artery; PCWP, pulmonary capillary wedge pressure; PH-HFpEF, pulmonary hypertension associated with heart failure with preserved ejection fraction; PVR, pulmonary vascular resistance. ${ }^{*} P<0.05$ for effect of inhaled NO (iNO) or nitrite compared to baseline (mixed-effects model); $\ddagger P<0.05$ for effect of nitrite compared to effect of inhaled NO (Hausman specification test).

lar decreases in right atrial pressure and pulmonary artery systolic, diastolic, and mean pressures. Cardiac index decreased slightly ( $<10 \%$ of baseline values) in PH-HFpEF and Group $1 \mathrm{PAH}$ patients but not in Group 3. PVR only decreased in Group 3. Cpa increased in PH-HFpEF and Group 3 PH but not Group 1 PAH. Changes in systemic blood pressures were similar across all groups; there were no significant changes in peripheral oxygen saturation.

Comparison of effects of inhaled sodium nitrite with inhaled NO. The effects of nitrite were significantly 
Table 2. Baseline echocardiographic data of the cohorts

\begin{tabular}{lcccc}
\hline & PH-HFpEF & Group 1 PAH & Group 3 PH & P \\
LVEDD (mm) & $49.7(45.5-54.1)$ & $47.8(42.4-51.9)$ & $45.8(40.8-50.8)$ & 0.46 \\
LVEDV (ml) & $110.9(88.9-127.4)$ & $99.1(81.0-116.0)$ & $76.8(67.1-98.8)$ & 0.63 \\
LVESV (ml) & $41.4(26.5-46.4)$ & $32.9(24.4-45.1)$ & $24.6(20.3-36.5)$ & 0.10 \\
LVEF (\%) & $65.1(58.7-71.1)$ & $65.1(61.1-70.0)$ & $68.2(61.1-71.5)$ & 0.70 \\
LA diameter (mm) & $40.3(37.7-42.9)$ & $36.4(34.0-37.1)$ & $36.1(34.5-38.8)$ & \\
RV FAC (\%) & $33.3(32.7-55.2)$ & $29.8(24.5-34.2)$ & $29.8(24.3-40.5)$ & 0.01 \\
TAPSE (mm) & $20.3(16.9-22.8)$ & $18.9(15.8-22.6)$ & $19.7(14.2-27.6)$ & 0.09 \\
\hline
\end{tabular}

Data displayed as median (interquartile range). LVEDD, left ventricular end-diastolic diameter; LVEDV, left ventricular end-diastolic volume; LVEF, left ventricular ejection fraction; LVESV, left ventricular end-systolic volume; LA, left atrium; RV FAC, right ventricular fractional area change; TAPSE, tricuspid annular plane systolic excursion.

greater than the effects of inhaled NO (iNO) for lowering right atrial pressure ( $-8.1 \mathrm{vs} .-0.4 \mathrm{mmHg}$, nitrite and iNO, respectively, $P<0.001)$ and pulmonary capillary wedge pressure $(-7.5 \mathrm{vs}$. $-0.6 \mathrm{mmHg}$, nitrite and iNO, respectively, $P<0.001$ ), mostly notable for PH-HFpEF (Supplemental Table 4). Mean pulmonary artery pressure was lowered more in $\mathrm{PH}-\mathrm{HFpEF}$ by inhaled nitrite than iNO $(-7.9$ vs. $-4.3 \mathrm{mmHg}$, nitrite and iNO, respectively, $P<0.001)$, while iNO had a greater effect on mean pulmonary artery pressure in Group $1 \mathrm{PAH}(-0.9$ vs. $-5.2 \mathrm{mmHg}$, nitrite and iNO, respectively, $P<0.001)$. Transpulmonary gradient was lowered more by iNO in all patients. PVR was decreased more by iNO in PH-HFpEF and Group 1 , but lowered similarly to the effect of nitrite in Group 3 patients. Cpa was increased more by nitrite in $\mathrm{PH}-\mathrm{HFpEF}$, while iNO increased Cpa more in Group 1, with an equivalent effect in Group 3. Figures 2-4 illustrate the effect of iNO as compared with nitrite in $\mathrm{PH}-\mathrm{HFpEF}$, Groups 1 and 3, respectively.

Resistance-compliance relationship. Administration of inhaled nitrite induced a preferential change in Cpa as compared with PVR, most notable in PH-HFpEF and Group $3 \mathrm{PH}$, but not Group $1 \mathrm{PAH}$. The change in compliance and not resistance is illustrated by the resistance-compliance curves (Figure 5), in which the data were fit to an inverse hyperbolic curve. There was no difference in the baseline resistance-compliance relationship between patient groups $(P=0.98)$. There was a trend towards shifting of the resistance-compliance curve up and to the right with administration of inhaled nitrite to patients with PH-HFpEF (Figure 5, $P=0.14$ ).

\section{Discussion}

In this study of the hemodynamic effects of inhaled sodium nitrite in patients with $\mathrm{PH}$, there were no significant safety concerns, satisfying the primary safety outcome of this phase IIa study. The most notable

Table 3. Baseline hemodynamics of the cohorts

\begin{tabular}{lcccc}
\hline & PH-HFpEF & Group 1 PAH & Group 3 PH & P \\
Heart Rate & $70(61-74)$ & $69(62-75)$ & $76(66-79)$ & 0.73 \\
RAP & $11.5(9.75-13.0)$ & $7.5(3.5-12.3)$ & $7.5(6.0-10.5)$ & 0.25 \\
mPAP & $35.7(31.3-41.6)$ & $45.2(31.3-48.1)$ & $36.2(34.8-47.5)$ & 0.38 \\
PCWP & $19.0(16.0-22.3)$ & $11.5(7.0-14.0)$ & $13.5(12.0-15.0)$ & $<.001$ \\
TPG & $14.0(13.2-21.6)$ & $33.5(22.0-37.6)$ & $23.7(20.5-32.8)$ & 0.001 \\
CI & $2.7(2.3-3.1)$ & $2.9(2.3-3.1)$ & $3.2(2.4-3.5)$ & 0.54 \\
PVR & $2.78(2.24-3.46)$ & $5.25(4.10-8.51)$ & $4.09(3.20-5.89)$ & 0.01 \\
Cpa & $2.50(2.07-4.40)$ & $1.74(1.28-2.39)$ & $2.06(1.22-2.98)$ & 0.003 \\
MAP & $94(77-105)$ & $84(76-93)$ & $91(82-103)$ & 0.15 \\
Csys & $1.30(1.14-1.63)$ & $1.62(1.20-2.14)$ & $1.41(0.99-2.02)$ & \\
SVR & $13.8(9.7-18.6)$ & $15.3(11.4-18.4)$ & $13.9(12.6-15.7)$ & 0.22 \\
\hline
\end{tabular}

Data displayed as median (interquartile range). RAP, right atrial pressure; mPAP, mean pulmonary artery pressure; PCWP, pulmonary capillary wedge pressure; TPG, transpulmonary gradient; CI, cardiac index; PVR, pulmonary vascular resistance; Cpa, pulmonary arterial compliance; MAP, mean arterial pressure; Csys, systemic arterial compliance; SVR, systemic vascular resistance. 

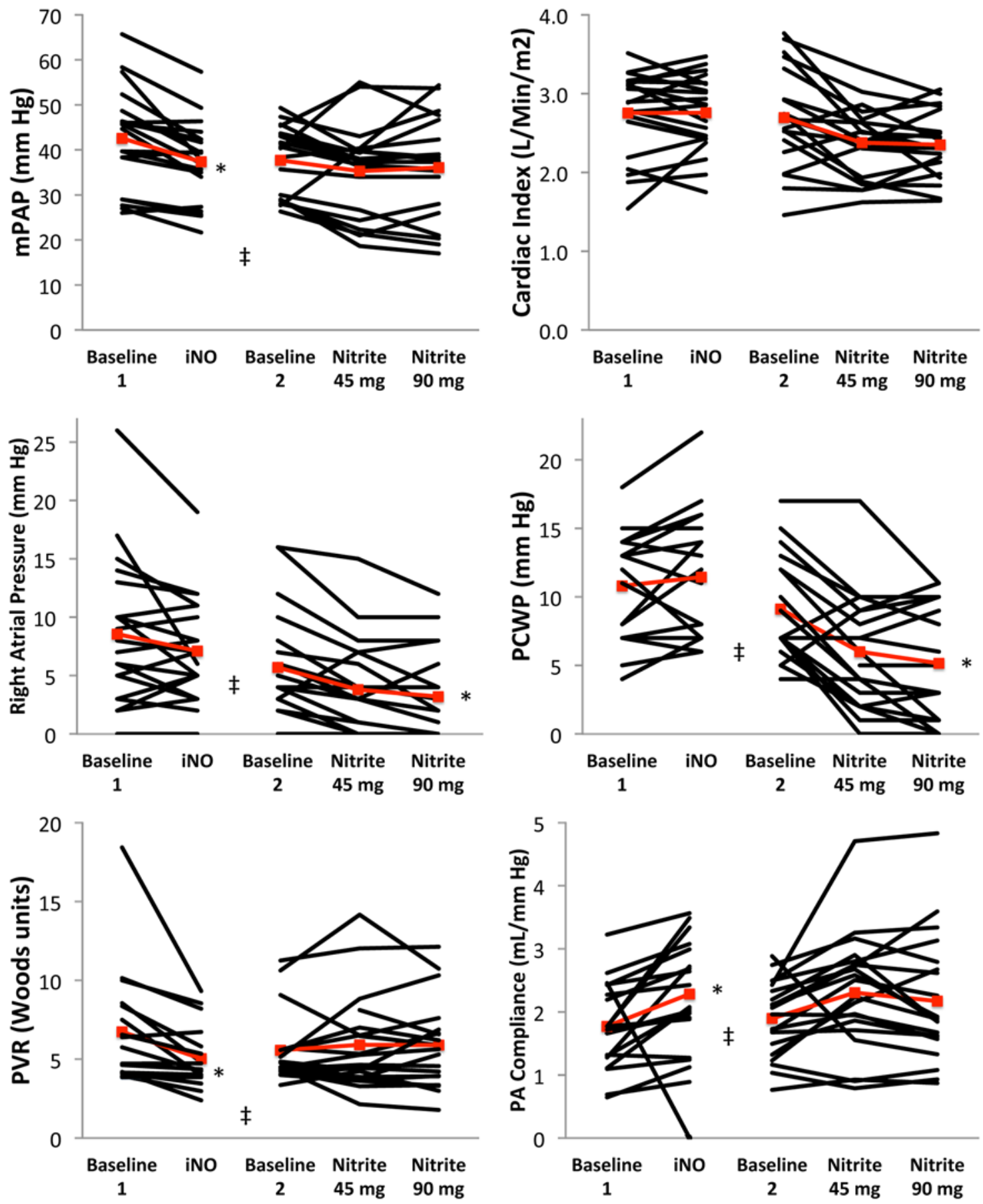

Figure 3. Effect of inhaled nitric oxide (iNO) and aerosolized sodium nitrite on Group 1 PAH patients. mPAP, mean pulmonary artery pressure; PA, pulmonary artery; $\mathrm{PAH}$, pulmonary arterial hypertension; PCWP, pulmonary capillary wedge pressure; PVR, pulmonary vascular resistance. ${ }^{*} P<0.05$ for effect of iNO or nitrite compared with baseline (mixed-effects model); $¥ P<0.05$ for effect of nitrite compared to effect of inhaled NO (Hausman specification test).

hemodynamic effect of inhaled sodium nitrite was the substantial decrease in pulmonary capillary wedge pressure and pulmonary artery pressures, with an increase in Cpa in PH-HFpEF patients. The acute lowering of filling pressures was distinctly different from the effect of iNO. PVR was modestly decreased in WHO Group $3 \mathrm{PH}$; however, there was no significant change in PH-HFpEF or Group $1 \mathrm{PAH}$, owing to significant decreases in both filling and pulmonary pressures resulting in no change in transpulmonary gradient. Administration of drug to patients appeared to be well tolerated.

Put in context of reports of increased functional capacity with administration of various other forms 

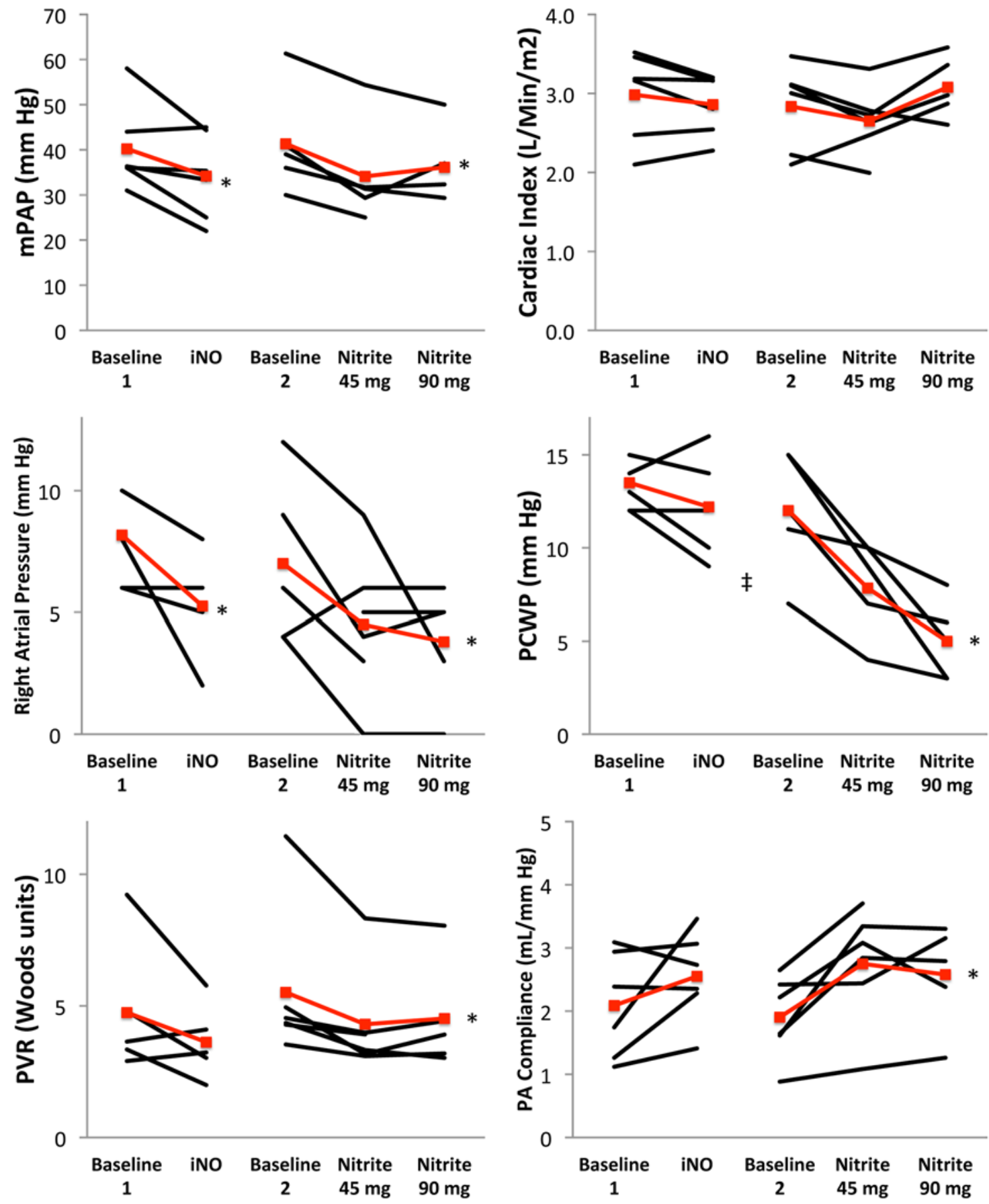

Figure 4. Effect of inhaled nitric oxide (iNO) and aerosolized sodium nitrite on Group 3 PH patients. mPAP, mean pulmonary artery pressure; PA, pulmonary artery; $\mathrm{PH}$, pulmonary hypertension; PCWP, pulmonary capillary wedge pressure; $\mathrm{PVR}$, pulmonary vascular resistance. ${ }^{*} P<0.05$ for effect of iNO or nitrite compared with baseline (mixed-effects model); $¥ P<0.05$ for effect of nitrite compared to effect of inhaled NO (Hausman specification test).

of nitrite (intravenous sodium nitrite and inorganic nitrate, which is in beet juice and is converted to nitrite by oral commensal bacteria) $(26,27)$, this report adds to our knowledge base of the physiologic effects of exogenous administration of nitrite in $\mathrm{PH}$ due to left heart disease, lung disease, and $\mathrm{PAH}$. Our data provide further evidence that inhaled nitrite lowers left- and right-sided filling pressures in patients with heart failure with preserved ejection fraction. This is likely to result in improvements in functional/exercise capacity and exertional dyspnea, which have been associated with higher baseline filling pressures (28). Two studies recently reported on the effects of a single intravenous infusion of sodium nitrite on hemodynamics at rest and/or with exercise in patients with $\mathrm{HFpEF}$, showing decreases in resting pulmonary artery and 
PH-HFpEF

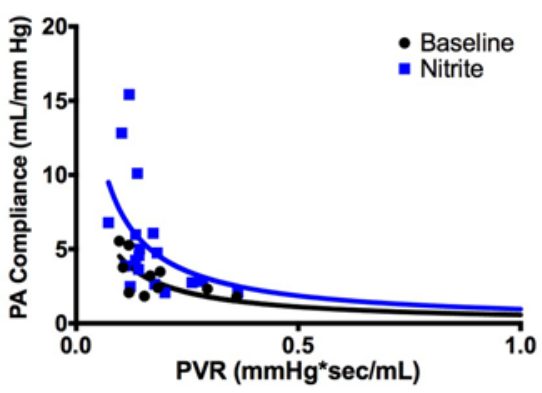

$P=0.14$
Group 1 PAH

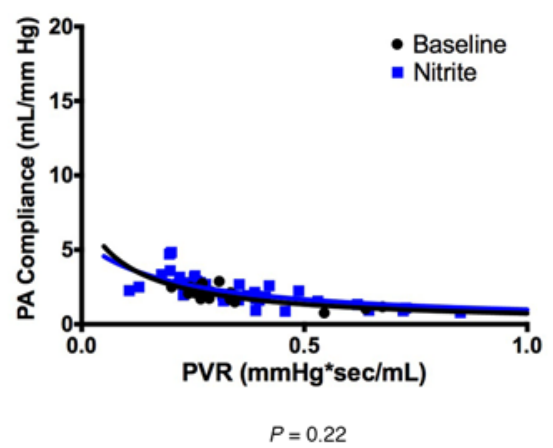

Group 3 PH

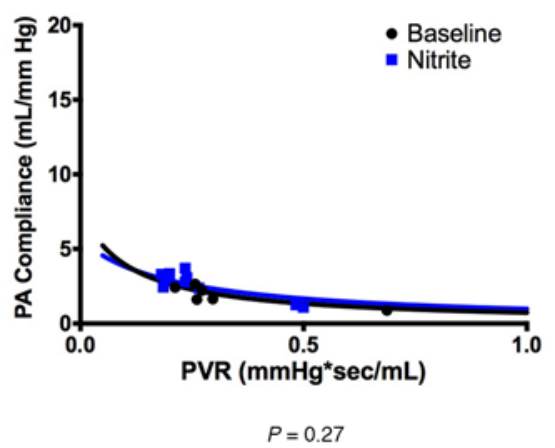

Figure 5. Resistance-compliance relationship at baseline and after aerosolized sodium nitrite. Data were fit to an inverse hyperbolic model with the following equations, in which $C$ is pulmonary artery compliance and $R$ is pulmonary vascular resistance: $\mathrm{PH}-\mathrm{HFpEF}$ baseline, $C=0.59 /(0.03+R), r^{2}=0.44$; PH-HFpEF nitrite, $C=0.99 /(0.03+R), r^{2}=0.25$; Group 1 PAH baseline, $C=0.81 /(0.10+R), r^{2}=0.61$; Group 1 PAH nitrite, $C=1.18 /(0.21+R), r^{2}=0.49 ;$ Group $3 \mathrm{PH}$ baseline, $C=0.63 /(0.04+R), r^{2}=0.71$; Group $3 \mathrm{PH}$ nitrite, $C=0.88 /(0.09+R), r^{2}=0.69$. The resistance-compliance relationship was not significantly altered in any group, although there was a trend in PH-HFpEF $(P=0.14$; Group $1 P=0.22$; Group $3 P=0.27$; extra sum-of-squares $\mathrm{F}$ test). $\mathrm{PH}-\mathrm{HFpEF}$, pulmonary hypertension associated with heart failure with preserved ejection fraction; $\mathrm{PAH}$, pulmonary arterial hypertension; $\mathrm{PH}$, pulmonary hypertension; $\mathrm{PA}$, pulmonary artery; PVR, pulmonary vascular resistance.

filling pressures without a change in PVR or Cpa and a marked blunting of the rise in pulmonary capillary wedge pressure with exercise $(22,29)$. A subsequent study has since been published by Borlaug and colleagues looking at the acute effects of inhaled nitrite on exercise hemodynamics, finding improvements in pulmonary capillary wedge pressure at rest as well as with exercise in patients with HFpEF. Other improvements seen were in mean pulmonary artery pressure, right atrial pressure, and Cpa. Our study extends the findings of improvements in resting hemodynamics, particularly filling pressures and Cpa, with inhaled nitrite to patients specifically with $\mathrm{PH}$ in the setting of HFpEF. Further, we provide data on a second and lower dose, $45 \mathrm{mg}$, which also had significant effects in a dose-dependent manner, which may allow future trials to incorporate dosing flexibility. Finally, in this trial we are able to compare responses in Group $2 \mathrm{PH}$ patients with those in Group 1 and $3 \mathrm{PH}$. Interestingly, we found improvements in Cpa, which may be due to inhaled as opposed to intravenous administration, differences in dosing or effective dose at the pulmonary vasculature, and/or different patient populations (our enrollment criteria required hemodynamic evidence of $\mathrm{PH})$.

Cpa increased in PH-HFpEF and Group 3 PH but not Group 1 PAH, and the PVR was unchanged in all groups, except Group 3. Thus, inhaled sodium nitrite's major physiological effect appears to be exerted on total pulmonary vascular compliance and not limited to pulmonary resistance vessels. Our data show that nitrite improves Cpa but not PVR. If we assume a fixed inverse hyperbolic relationship between Cpa and PVR $(30,31)$, then hemodynamic response to nitrite can only occur in those patients who fall along the upper portion of the shoulder of the resistance-compliance curve. This portion of the resistance-compliance curve is where large changes in compliance can occur with little change in resistance, e.g., PH-HFpEF patients. Group 3 patients fall more in the middle of the shoulder of the resistance-compliance curve and thus exhibit a more modest hemodynamic response to nitrite. Group $1 \mathrm{PAH}$ patients are on the far right flat portion of the resistance-compliance curve (high PVR, low Cpa) where compliance cannot change without a large change in resistance, explaining the lack of effect of nitrite in Group 1 PAH. This observation provides further physiological support for the role of exogenous administration of sodium nitrite for nonGroup $1 \mathrm{PH}$ patients. Tedford et al. demonstrated that elevated pulmonary capillary wedge pressure was associated with lower Cpa for any given PVR (shifting the resistance-compliance curve down and to the left), although there is still some debate about this in the literature $(25,32)$. We found no difference in the baseline resistance-compliance relationship between patient groups. However, there was a trend towards shifting of the resistance-compliance curve up and to the right with administration of inhaled nitrite, suggesting that if the resistance-compliance relationship is indeed altered by left ventricular filling pressure, then exogenous administration of nitrite may have an additional beneficial physiological effect.

Our study has several limitations. It was a small, early phase II pilot study and therefore involved limited numbers of patients. As this trial's inception predated any safety information, it was designed 


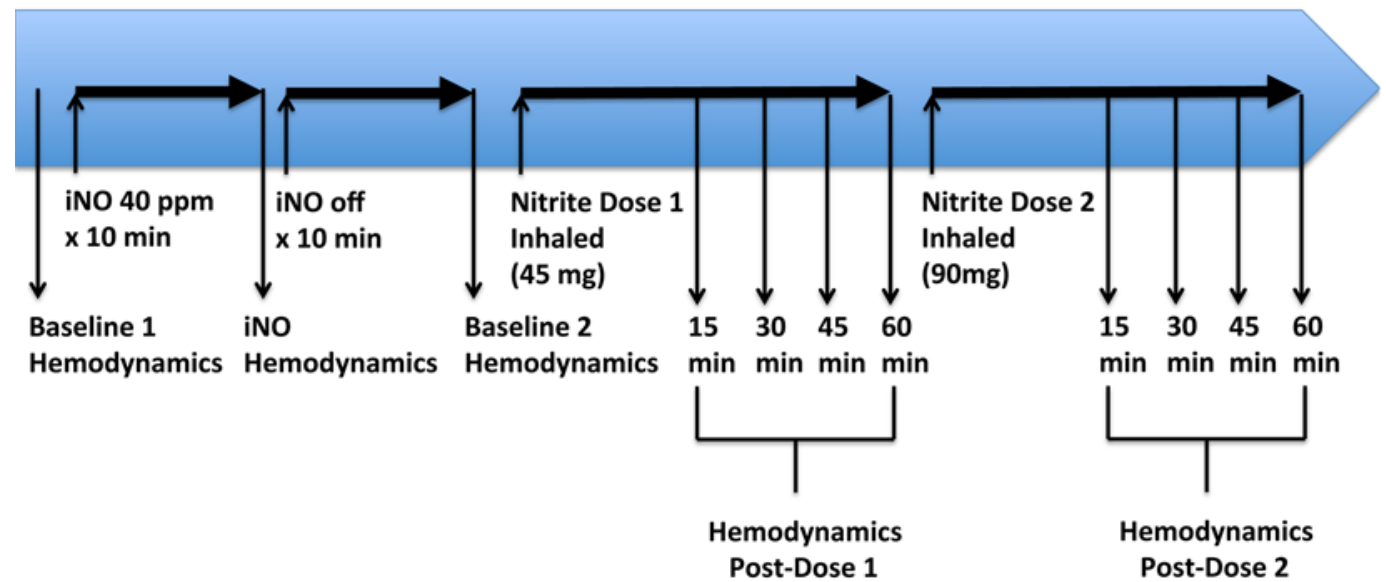

Figure 6. Protocol design. Baseline hemodynamics (baseline 1) were initially recorded followed by administration of inhaled nitric oxide (iNO) for 10 minutes, at which time hemodynamics were recorded again. NO was then turned off, allowed to wear off for 10 minutes, and then baseline hemodynamics (baseline 2) were recorded. Next, aerosolized sodium nitrite was administered at the first dose of $45 \mathrm{mg}$ (dose 1). Hemodynamics were recorded 15 minutes after completion of the inhaled dose, and then at 30, 45, and 60 minutes after completion of dose 1. Dose 2 ( 90 mg) of aerosolized sodium nitrite was administered next. Hemodynamics were recorded 15 minutes after completion of the inhaled dose, and then at 30,45 , and 60 minutes after completion of dose 2 .

primarily as a safety trial, and therefore exercise hemodynamics were not a part of the trial design, which would be valuable given the exertional symptoms of $\mathrm{PH}-\mathrm{HFpEF}$ patients. These findings of acute changes in hemodynamics may or may not be translatable to chronic administration and longer-term outcomes.

In conclusion, we find that inhaled sodium nitrite appears to be safe in Groups 1-3 $\mathrm{PH}$ and may be efficacious in Groups 2 and $3 \mathrm{PH}$, primarily via improvements in Cpa. Further study in non-Group $1 \mathrm{PH}$ is warranted.

\section{Methods}

Study setting. This was a prospective open-label safety and efficacy trial. Safety endpoints included change in systemic blood pressure and methemoglobin levels. Efficacy endpoints were changes in invasively measured cardiopulmonary hemodynamics. Given the physiological differences between PH-HFpEF, WHO Group $1 \mathrm{PAH}$ and Group $3 \mathrm{PH}$, as well as the relative lack of knowledge of the pulmonary vascular biology in $\mathrm{PH}-\mathrm{HFpEF}$, the efficacy endpoints were exploratory for PH-HFpEF. The primary efficacy outcome was prospectively defined for Group $1 \mathrm{PAH}$ and Group $3 \mathrm{PH}$ as the change in PVR. Secondary endpoints included changes in $\mathrm{Cpa}$, pulmonary, right atrial, and pulmonary capillary wedge pressures. Patients with PH-HFpEF, WHO Group $1 \mathrm{PAH}$, or Group $3 \mathrm{PH}$ were enrolled. PH-HFpEF patients were enrolled if pulmonary capillary wedge pressure was greater than $15 \mathrm{mmHg}$ and transpulmonary gradient greater than $12 \mathrm{mmHg}$ in the setting of left ventricular ejection fraction (LVEF) greater than or equal to $40 \%$ and dyspnea resulting in at least a mild limitation on physical activity (functional class II or greater). WHO Group 1 $\mathrm{PAH}$ patients (idiopathic $\mathrm{PAH}$ or associated with connective tissue disease, or due to drug exposure) were required to be on stable therapy for at least 3 months. WHO Groups $1 \mathrm{PAH}$ and $3 \mathrm{PH}$ (due to parenchymal lung disease) patients were enrolled if PVR was greater than or equal to 3 Woods units. Hemodynamic

Table 4. Hemodynamic criteria for enrollment based upon PH group

\begin{tabular}{|c|c|c|c|}
\hline & PH-HFpEF & Group 1 PAH & Group 3 PH \\
\hline MPAP (mmHg) & $\geq 25$ & $\geq 25$ & $\geq 25$ \\
\hline PCWP (mmHg) & $>15$ & $\leq 15$ & $\leq 15$ \\
\hline TPG (mmHg) & $>12$ & - & - \\
\hline PVR (Woods units) & - & $\geq 3$ & $\geq 3$ \\
\hline
\end{tabular}

MPAP, mean pulmonary artery pressure; PCWP, pulmonary capillary wedge pressure; PH, pulmonary hypertension; PH-HFpEF, pulmonary hypertension associated with heart failure with preserved ejection fraction; PVR, pulmonary vascular resistance; TPG, transpulmonary gradient. 
criteria for enrollment are summarized in Table 4. Other key inclusion criteria included a mean pulmonary artery pressure greater than or equal to $25 \mathrm{mmHg}$, pulmonary capillary wedge pressure less than or equal to $15 \mathrm{mmHg}$ (for Groups 1 and 3), and ages 18-78. Key exclusion criteria included baseline systemic hypotension (mean arterial pressure $<50 \mathrm{mmHg}$ ) or hypertension (a systolic blood pressure $>160 \mathrm{mmHg}$ or diastolic blood pressure $>100 \mathrm{mmHg}$ ), use of intravenous inotropes within 30 days, a history of portal hypertension or chronic liver disease, renal insufficiency with a serum creatinine greater than $2.5 \mathrm{mg} / \mathrm{dl}$, anemia with hemoglobin less than $9 \mathrm{~g} / \mathrm{d}$, symptomatic coronary atherosclerotic disease, history of red blood cell CYP B5 reductase deficiency or glucose-6-phosphate dehydrogenase deficiency, and females with a positive pregnancy test or actively breast feeding.

Aerosolized sodium nitrite. Sodium nitrite (AIR001 Inhalation Solution, Aires Pharmaceuticals, Inc., a subsidiary of Mast Therapeutics, Inc.) was supplied in an aseptically filled blow-fill-sealed ampule containing $80 \mathrm{mg} / \mathrm{ml}$ in a sterile phosphate buffer solution. Phase I pharmacokinetics and pharmacodynamics of dosing have been published $(18,24)$. To maintain matching osmolarity, doses for the study were diluted to a final concentration of $11.25 \mathrm{mg} / \mathrm{ml}$ with $0.9 \%$ normal saline for injection to a final volume of $4 \mathrm{ml}$ for the $45 \mathrm{mg}$ dose, and to a final concentration $22.5 \mathrm{mg} / \mathrm{ml}$ with sterile water for injection to a final volume of $4 \mathrm{ml}$ for the $90 \mathrm{mg}$ dose. Doses of $45 \mathrm{mg}$ and $90 \mathrm{mg}$ (the amount placed into the nebulizer medication chamber) were administered via the Aerogen Solo-Idehaler nebulization device, which is composed of the nebulization head (Aeroneb Solo, Aerogen) and the aerosol-reservoir attachment (Idehaler, Diffusion Technique Francais). The electronic nebulizer delivers the dose at high efficiency (approximately 65\%) over 10 to 12 minutes. The dose of $90 \mathrm{mg}$ has previously been reported as the maximally tolerated dose $(18,24)$.

Protocol. After obtaining informed consent, patients underwent a standard right heart catheterization via the right internal jugular vein, followed by vasodilator challenge with $40 \mathrm{ppm}$ iNO. If patients met hemodynamic criteria (Table 4), then aerosolized sodium nitrite was administered at the first dose of 45 mg. Invasive cardiopulmonary hemodynamics (right atrial pressure, pulmonary artery pressure, pulmonary capillary wedge pressure, and cardiac output) were recorded every 15 minutes for 1 hour. Figure 6 illustrates the protocol. Of note, right ventricular pressures were only recorded at baseline and at the final time point at end of study so as to minimize manipulation of the pulmonary artery catheter. If stopping criteria were not met (decrease in systemic systolic blood pressure $>40 \mathrm{mmHg}$, decrease in peripheral oxygen saturation $>10 \%$, methemoglobin level $>5 \%$, severe bronchospasm or dyspnea), then a second dose of $90 \mathrm{mg}$ aerosolized sodium nitrite was administered. Invasive cardiopulmonary hemodynamics were recorded every 15 minutes for 1 hour after this second dose. Systemic blood pressure, peripheral oxygen saturation, and methemoglobin levels were continuously monitored throughout the procedure. All patients were instructed by the research RN (PMW) prior to drug administration to report any symptoms, and all patients were reminded by the investigator (MAS, present during entire procedure) after drug administration to report symptoms.

Right heart catheterization. Right heart catheterization was performed using a balloon-tipped thermodilution 7F Swan-Ganz catheter for the measurements of hemodynamics. Cardiac output was recorded as the average of at least 3 thermodilution measurements. Transpulmonary gradient was calculated as (mean pulmonary artery pressure - pulmonary capillary wedge pressure); PVR as transpulmonary gradient/cardiac output; total pulmonary resistance as mean pulmonary artery pressure/cardiac output; Cpa as stroke volume/pulse pressure.

Statistics. Variable distributions were evaluated for normality and transformed as needed. Baseline data are expressed as median values, with interquartile ranges. We first compared the distribution of study outcome variables between the 3 patient groups (WHO Groups 1,2, and 3) before aerosolized sodium nitrite intervention using the nonparametric Kruskal-Wallis test. To test the effect of aerosolized sodium nitrite on hemodynamic parameters at all time points relative to baseline (15, 30, 45, and 60 minutes after dose 1; and 15, 30, 45 and 60 minutes after dose 2), a linear mixed-effect model was used. In this model, the effect of treatment on hemodynamics (15-60 minutes after dose) was compared with baseline values. We assessed the overall linear trend of treatment. The effect of treatment on hemodynamics in each patient group was assessed separately in mixed-effects models. The interaction between treatment and patient group was tested and corresponding $P$ values reported. Finally, the effect of aerosolized sodium nitrite dose (45 and $90 \mathrm{mg}$ ) on hemodynamic changes within each patient group was tested in mixed-effects models. For iNO, we used mixed-effect models to assess the effect of iNO versus baseline; this was done for consistency of analysis between effects of iNO and effects of nitrite. We tested the coefficients in the 2 aforementioned 
models (model assessing the effect of iNO vs. model assessing the effect of aerosolized sodium nitrite) by the Hausman specification test to assess if the effects of the 2 agents are similar or not. Treatment effects are presented as mean values and their $95 \%$ confidence intervals. Resistance-compliance curves were constructed and the data were fit to an inverse hyperbolic curve and compared using the extra sum-of-squares F test (Prism 6, GraphPad Software) (25). Statistical significance was defined as a 2-sided $P$ value less than 0.05. All statistical calculations, except as noted for the resistance-compliance relationship, were made using STATA 14.0 (StataCorp).

Study approval. The protocol was approved by the University of Pittsburgh Institutional Review Board, is registered with the Food and Drug Administration as an investigative new drug protocol, and is listed on clinicaltrials.gov (NCT01431313). All participants signed written informed consent.

\section{Author contributions}

MAS, ELP, and MTG: study design. MAS and PMW: patient recruitment, care and follow-up. MAS, RRV, TNB, and PMW: data collection, maintenance, and analysis. MS and JG: analysis of echocardiographic data. MAS and MN: statistical analysis. MAS, RRV, MN, TNB, PMW, MS, JG, ELP, and MTG: critical revision of the manuscript for important intellectual content. MAS had access to the study data and takes full responsibility for its integrity and accuracy.

\section{Acknowledgments}

Sources of Funding: This work supported in part by the NIH grants 2P01HL103455 (to MAS and MTG), R01HL098032 (to MTG), R01HL096973 (to MTG), and Mast Therapeutics, Inc. Inhaled sodium nitrite and nebulizer equipment were supplied by Mast Therapeutics, Inc.

Address correspondence to: Marc A. Simon, Associate Professor of Medicine, Heart and Vascular Institute, University of Pittsburgh, Scaife Hall S-555, 200 Lothrop Street, Pittsburgh, Pennsylvania 15213, USA. Phone: 412.802.3131; E-mail: simonma@upmc.edu.

1. Vachiéry JL, et al. Pulmonary hypertension due to left heart diseases. J Am Coll Cardiol. 2013;62(25 Suppl):D100-D108.

2. Guazzi M, Borlaug BA. Pulmonary hypertension due to left heart disease. Circulation. 2012;126(8):975-990.

3. Miller WL, Grill DE, Borlaug BA. Clinical features, hemodynamics, and outcomes of pulmonary hypertension due to chronic heart failure with reduced ejection fraction: pulmonary hypertension and heart failure. JACC Heart Fail. 2013;1(4):290-299.

4. Lam CS, Roger VL, Rodeheffer RJ, Borlaug BA, Enders FT, Redfield MM. Pulmonary hypertension in heart failure with preserved ejection fraction: a community-based study. J Am Coll Cardiol. 2009;53(13):1119-1126.

5. Califf RM, et al. A randomized controlled trial of epoprostenol therapy for severe congestive heart failure: The Flolan International Randomized Survival Trial (FIRST). Am Heart J. 1997;134(1):44-54.

6. Packer M, et al. Clinical effects of endothelin receptor antagonism with bosentan in patients with severe chronic heart failure: results of a pilot study. J Card Fail. 2005;11(1):12-20.

7. Guazzi M, Vicenzi M, Arena R, Guazzi MD. Pulmonary hypertension in heart failure with preserved ejection fraction: a target of phosphodiesterase-5 inhibition in a 1-year study. Circulation. 2011;124(2):164-174.

8. Redfield MM, et al. Effect of phosphodiesterase-5 inhibition on exercise capacity and clinical status in heart failure with preserved ejection fraction: a randomized clinical trial. JAMA. 2013;309(12):1268-1277.

9. Wu X, Yang T, Zhou Q, Li S, Huang L. Additional use of a phosphodiesterase 5 inhibitor in patients with pulmonary hypertension secondary to chronic systolic heart failure: a meta-analysis. Eur J Heart Fail. 2014;16(4):444-453.

10. Galiè N, Humbert M, Vachiery JL, Gibbs S, Lang I, Torbicki A, et al. 2015 ESC/ERS Guidelines for the diagnosis and treatment of pulmonary hypertension: The Joint Task Force for the Diagnosis and Treatment of Pulmonary Hypertension of the European Society of Cardiology (ESC) and the European Respiratory Society (ERS): Endorsed by: Association for European Paediatric and Congenital Cardiology (AEPC), International Society for Heart and Lung Transplantation (ISHLT) [published correction appears in Eur Respir J. 2015;46(6):1855-1856]. Eur Respir J. 2015;46(4):903-975.

11. Naeije R, Vachiery JL, Yerly P, Vanderpool R. The transpulmonary pressure gradient for the diagnosis of pulmonary vascular disease. Eur Respir J. 2013;41(1):217-223.

12. Gerges C, et al. Diastolic pulmonary vascular pressure gradient: a predictor of prognosis in "out-of-proportion" pulmonary hypertension. Chest. 2013;143(3):758-766.

13. Tampakakis E, et al. The diastolic pulmonary gradient does not predict survival in patients with pulmonary hypertension due to left heart disease. JACC Heart Fail. 2015;3(1):9-16.

14. Gerges M, et al. Pulmonary hypertension in heart failure. epidemiology, right ventricular function, and survival. Am J Respir Crit Care Med. 2015;192(10):1234-1246.

15. Vanderpool RR, Gladwin MT, Simon MA. Hemodynamic markers of pulmonary vascular disease in pulmonary hypertension due to left heart disease. J Heart Lung Transplant. 2015;34:S118.

16. Humbert M, Sitbon O, Simonneau G. Treatment of pulmonary arterial hypertension. N Engl J Med. 2004;351(14):1425-1436. 
17. Lundberg JO, Weitzberg E, Gladwin MT. The nitrate-nitrite-nitric oxide pathway in physiology and therapeutics. Nat Rev Drug Discov. 2008;7(2):156-167.

18. Rix PJ, et al. Pharmacokinetics, pharmacodynamics, safety, and tolerability of nebulized sodium nitrite (AIR001) following repeat-dose inhalation in healthy subjects. Clin Pharmacokinet. 2015;54(3):261-272.

19. Cosby K, et al. Nitrite reduction to nitric oxide by deoxyhemoglobin vasodilates the human circulation. Nat Med. 2003;9(12):1498-1505

20. Hunter CJ, et al. Inhaled nebulized nitrite is a hypoxia-sensitive NO-dependent selective pulmonary vasodilator. Nat Med 2004;10(10):1122-1127.

21. Zuckerbraun BS, et al. Nitrite potently inhibits hypoxic and inflammatory pulmonary arterial hypertension and smooth muscle proliferation via xanthine oxidoreductase-dependent nitric oxide generation. Circulation. 2010;121(1):98-109.

22. Borlaug BA, Koepp KE, Melenovsky V. Sodium nitrite improves exercise hemodynamics and ventricular performance in heart failure with preserved ejection fraction. J Am Coll Cardiol. 2015;66(15):1672-1682.

23. Lai YC, et al. SIRT3-AMP-activated protein kinase activation by nitrite and metformin improves hyperglycemia and normalizes pulmonary hypertension associated with heart failure with preserved ejection fraction. Circulation. 2016;133(8):717-731

24. Parsley EL, Masamune H, Hoye WL, Rubin LJ, Gladwin MT. P66: Nebulized inhaled nitrite (AIR001) for pulmonary arterial hypertension: Studies to determine safety, pharmacokinetics, and maximum tolerated dose, lack of pharmacodynamic interaction with sildenafil and optimal nebulizer device. Nitric Oxide. 2013;31(1):S41-S42.

25. Tedford RJ, et al. Pulmonary capillary wedge pressure augments right ventricular pulsatile loading. Circulation. 2012;125(2):289297.

26. Zamani P, et al. Effect of inorganic nitrate on exercise capacity in heart failure with preserved ejection fraction. Circulation 2015;131(4):371-80; discussion 380

27. Coggan AR, et al. Acute dietary nitrate intake improves muscle contractile function in patients with heart failure: a doubleblind, placebo-controlled, randomized trial. Circ Heart Fail. 2015;8(5):914-920.

28. Borlaug BA, Paulus WJ. Heart failure with preserved ejection fraction: pathophysiology, diagnosis, and treatment. Eur Heart J. 2011;32(6):670-679

29. Ormerod JO, et al. Short-term intravenous sodium nitrite infusion improves cardiac and pulmonary hemodynamics in heart failure patients. Circ Heart Fail. 2015;8(3):565-571.

30. Lankhaar JW, et al. Pulmonary vascular resistance and compliance stay inversely related during treatment of pulmonary hypertension. Eur Heart J. 2008;29(13):1688-1695.

31. Lankhaar JW, et al. Quantification of right ventricular afterload in patients with and without pulmonary hypertension. $A m J$ Physiol Heart Circ Physiol. 2006;291(4):H1731-H1737.

32. Chemla D, Lau EM, Papelier Y, Attal P, Hervé P. Pulmonary vascular resistance and compliance relationship in pulmonary hypertension. Eur Respir J. 2015;46(4):1178-1189. 